\title{
The geometric clutch at 20: stripping gears or gaining traction?
}

\author{
Charles B Lindemann and Kathleen A Lesich \\ Department of Biological Sciences, Oakland University, Rochester, Michigan 48309, USA \\ Correspondence should be addressed to C B Lindemann; Email: lindemann@oakland.edu
}

\begin{abstract}
It has been 20 years since the geometric clutch (GC) hypothesis was first proposed. The core principle of the GC mechanism is fairly simple. When the axoneme of a eukaryotic flagellum is bent, mechanical stress generates forces transverse to the outer doublets (t-forces). These $t$-forces can push doublets closer together or pry them apart. The GC hypothesis asserts that changes in the inter-doublet spacing caused by $t$-forces are responsible for the activation and deactivation of the dynein motors, that creates the beat cycle. A series of computer models utilizing the clutch mechanism has shown that it can simulate ciliary and flagellar beating. The objective of the present review is to assess where things stand with the GC hypothesis in the clarifying light of new information. There is considerable new evidence to support the hypothesis. However, it is also clear that it is necessary to modify some of the original conceptions of the hypothesis so that it can be consistent with the results of recent experimental and ultrastructural studies. In particular, dynein deactivation by $t$-forces must be able to occur with dyneins that remain attached to the B-subtubule of the adjacent doublet. Reproduction (2015) 150 R45-R53
\end{abstract}

\section{Introduction}

The mechanism by which eukaryotic cilia and flagella generate a beat cycle has been one of the most enduring puzzles in cell biology. Perhaps the reason the mechanism has been refractory to intuitive resolution is that the eukaryotic flagellum (cilium) spans two levels of complexity. The cilium is an intricate structure assembled from more than 250 known components (Dutcher 1995). In fact, a proteomic analysis identified more than 600 cilia-related proteins, and more than half were identified with high confidence (Pazour et al. 2005, Davis et al. 2006). Most of the component proteins are arranged on a geometrically intricate scaffold composed of nine outer doublets and two central microtubules. This complex structure, called the axoneme, forms the main skeleton of the cilium and is assembled from a basal body that is a nine-element centriole. To power a typical $6 \mu \mathrm{m}$ human epithelial cilium requires $\sim 7200$ dynein motor proteins. These proteins are arranged in two rows on all but one of the outer doublet microtubules, and at least 15 subtypes of axonemal dynein have been identified in Chlamydomonas flagella, each of which is encoded by a different gene (for reviews, see Hom et al. (2011), Kikkawa (2013), Kamiya \& Yagi (2014) and Oda et al. (2014)). An illustration of a flagellar axoneme in cross-section is shown in Fig. 1.

Each dynein motor protein is a large and complex molecular structure. The dynein motor has been the most difficult of the molecular motors to understand. The heavy chain of the motor is extremely large $(\sim 450000 \mathrm{Da})$ and extensively folded. The AAA domain that makes up the globular head of the motor has not one but four nucleotide binding sites. Most puzzling, the AAA1 nucleotide binding site, which powers the power stroke of the motor, is $\sim 25 \mathrm{~nm}$ removed from the tip of the coiled coil stalk that must transmit the force of the power stroke to the adjacent outer doublets. In addition to this almost bizarre degree of complexity, the heavy chain requires an extensive set of accessory light and intermediate chain proteins for full functionality (Gatti et al. 1989).

It is no wonder then that when the geometric clutch (GC) mechanism was first proposed in 1994, it was not given much credibility. The GC mechanism is almost absurdly reductionist in its simplicity. It focuses entirely on the mechanics of the scaffold created by the microtubular axoneme. It contends that when the dynein motors contact a microtubule, they generate a sliding motion between the doublets. This view of dynein was suggested by the early work on isolated flagellar dyneins. Paschal et al. (1987) first demonstrated that outer-arm dynein layered on glass would translocate microtubules upon contact in the presence of Mg-ATP. Of course, the motors must be in their active state and not inactivated by dephosphorylation. The GC hypothesis assumes that when these minimal conditions are met, the regulation of the motors is simply a matter of positioning. The GC 


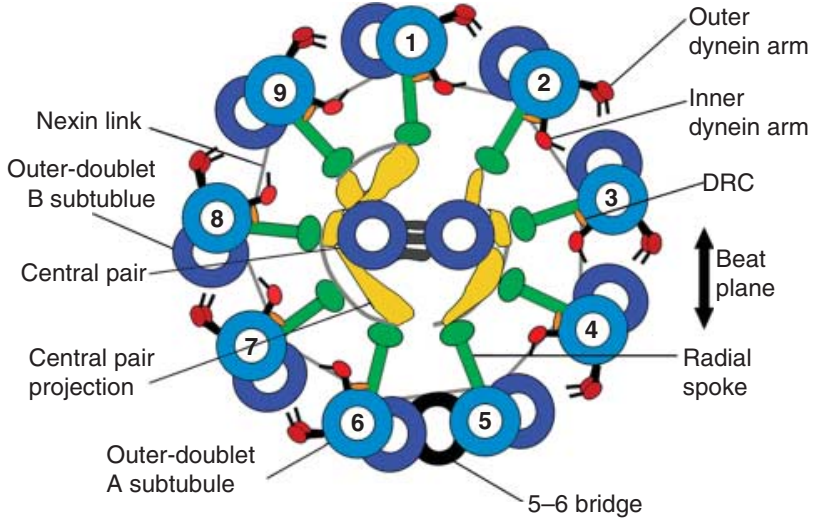

Figure $1 \mathrm{~A}$ schematic diagram of the axoneme of a eukaryotic cilium or flagellum as viewed in cross-section. The component parts are labeled for the convenience of the reader. Reproduced with permission from Lindemann, CB \& Lesich KA 2010. Flagellar and ciliary beating: the proven and the possible. Journal of Cell Science 123 519-528. (doi:10.1242/jcs.051326) (c) Company of Biologists.

hypothesis makes the assumption that during the relaxed state of the axoneme, dynein motors are not positioned close enough to engage the next doublet; they need to be closer together. By the same logic, the model contends that if the doublets are pulled away from each other, the dyneins will stop pulling.

The most important novel element contributed by the hypothesis is the recognition that when there is linear tension (or compression) on the outer doublets of a bending cilium (or flagellum), a force will always develop in the plane of the bend and transverse to the long axis of the doublet (t-force). The $t$-forces that develop within a cilium that is being bent by the action of the dynein motors will act to pry the interacting doublets apart, and they will therefore act to terminate the action of the motors. When the cilium is passively bent by fluid flow or by mechanically imposed bending, $\mathrm{t}$-force also develops, but this t-force causes a compression of the structure that pushes some of the doublets closer together. This relationship of t-force to active and passive bending is illustrated in Fig. 2.

In an intact cilium, the doublets are arranged in a circle. Therefore, the action of dyneins on one side can also be considered to act by passively bending the doublets on the opposite, inactive side. In this way, the dyneins on opposite sides may participate in their own inactivation while simultaneously ensuring the activation of the opposing set of dyneins on the other side of the axoneme. It is this geometric arrangement that provides for the reciprocal activation of opposing dynein sets and allows the GC mechanism to establish a repetitive bidirectional beat cycle.

The clutch mechanism is well defined and simple enough that it was amenable to modeling the working mechanism on a computer. The first model (Lindemann 1994a) demonstrated that it could produce a stable, reciprocating, cilia-like beat via the t-force mechanism, which inactivates the motors when the outwardly directed $t$-force reached a certain threshold and activates the motors when an inward (pinching) t-force reached a defined threshold. It also produces a flagellar-like bending wave if the structure is long enough and free to pivot at the base. This first computer model of the GC required a push-start; a straight flagellum has no curvature and hence no t-force to initiate the first activation of the motors.

Obviously, most real flagella do not require a pushstart. Some actually do, but that is another story. The second version of the clutch model (Lindemann 1994b) solved the startup problem by instilling a random probability of spontaneous attachment to each dynein motor. In subsequent iterations of the program, attached dyneins contribute a small positive $t$-force that increases
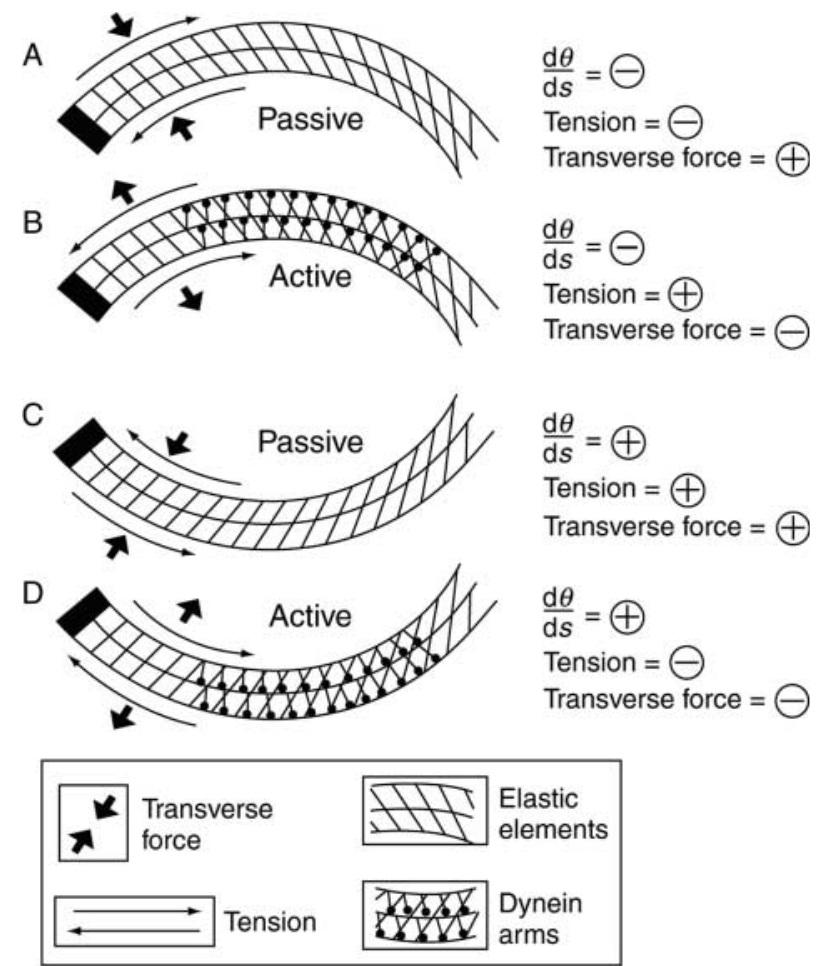

Figure 2 A diagram illustrating the relationship between transverse force ( $\mathrm{t}$-force) and bending. When two doublets that are linked to each other only by passive elastic linkages are bent ( $\mathrm{A}$ and $\mathrm{C}$ ), the tension on the doublets generated by the elastic linkers results in a pinching t-force that forces the doublets closer to each other. In contrast, when active motors bend the doublets ( $\mathrm{B}$ and $\mathrm{D})$, the tension on the doublets generated by the active motors results in an outwardly directed t-force that acts to pull the doublets apart. This relationship is independent of the direction of bending, as is shown in the diagram. The sign convention that was used for modeling the action of t-force is shown at the right of each doublet pair. Figure reproduced from Lindemann CB (2011) Experimental evidence for the geometric clutch hypothesis.

Current Topics in Developmental Biology 95 1-31 with permission and modified from Lindemann, CB 1994b A model of flagellar and ciliary functioning which uses the forces transverse to the axoneme as the regulator of dynein activation. Cell Motility and the Cytoskeleton 29 141-154 with permission. 


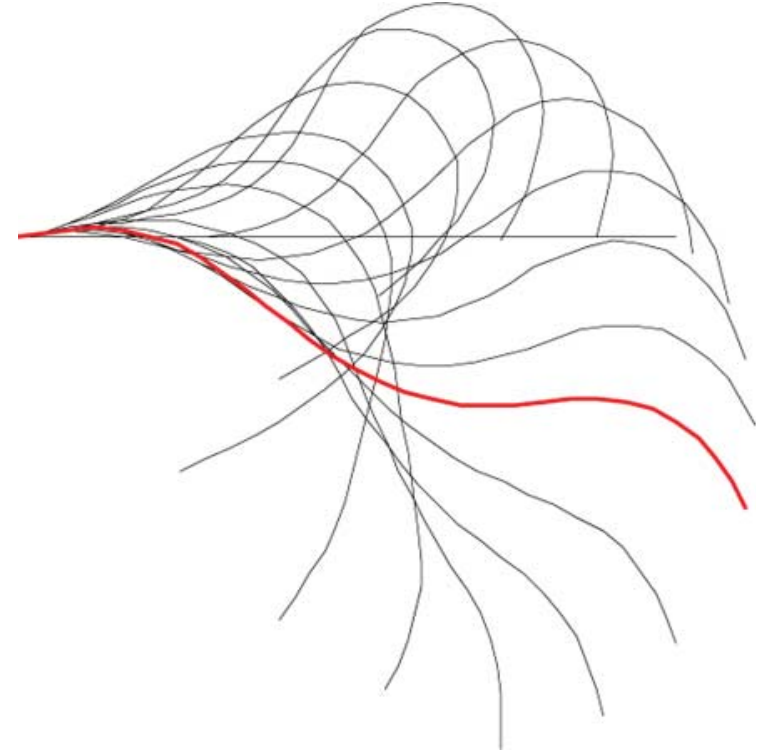

Figure 3 Geometric clutch simulation of a $12 \mu \mathrm{m}$-long cilium. The output of the geometric clutch computer model version 3 is shown for the last beat cycle of a 1500 iteration run. Every tenth iteration is plotted, and each iteration represents $0.1 \mathrm{~ms}$. This version of the geometric clutch model was used as the test platform for the study published in Lindemann (2002). The ciliary effective and recovery strokes are both produced by an identical modeling algorithm that was used to control the dyneins on both sides of the axoneme. The asymmetry of the beat results from assigning a different base level of probability of initial attachment to the dyneins on the two sides of the axoneme: 0.06 for the P-bend dyneins and 0.01 for the R-bend dyneins.

the probability of that neighboring dyneins will attach. This has the effect of generating a cascade of attachment somewhere on the cilium that gets things started. The second model also incorporated realistic values for the mechanical properties of the cilium and for drag resistance from the surrounding viscous medium. The force per dynein head and bending resistance of the cilium (or flagellum) were adapted from prior experimental estimates. The improved model that resulted simulated the behavior of cilia and flagella quite realistically and did not require a push-start. Output from that version of the model is shown in Fig. 3.

In the intervening years since the hypothesis was first developed, it has successfully duplicated the behavior of real cilia and predicted the behavior of real flagella. At the most basic level, if $t$-force is responsible for dynein switching, then preventing the development of $t$-force should interrupt the beat cycle. We tested this directly in bull sperm and showed that preventing the development of curvature by mechanically blocking the formation of a basal bend arrests the beat in an active, force-generating stall (Holcomb-Wygle et al. 1999, Schmitz et al. 2000). Shortening a bull sperm flagellum by clipping it to a length of $15 \mu \mathrm{m}$ (30\% of the total length) or less also stalls the beat at either endpoint of the beat cycle
(Holcomb-Wygle et al. 1999), as was predicted by the GC computer model (Lindemann \& Kanous 1995).

It has been well established by experimental evidence that extraction of the outer dynein arms reduces the beat frequency, with only a minor change in waveform of the beat (Brokaw \& Kamiya 1987). This experimental observation was initially a significant challenge for the GC model. A reduction in the number of functional dyneins generally caused a radical change in the waveform of the beat or the complete cessation of beating. Brokaw (1999) showed that in order for his curvature-controlled computer model to match the experimental result of outer-arm extraction, the inner and outer arms had to have considerably different forcevelocity behavior.

The major difference between the force-velocity profiles is that inner arms must contribute most of the force and inter-doublet adhesion at low sliding velocities, whereas the outer arms contribute more of the power at higher sliding velocities. When force-velocity behavior for inner- and outer-arm dyneins, similar to that employed by Brokaw, was incorporated into the GC model, it allowed for the conservation of waveform after the outer arms were disabled (Lindemann 2002). This suggests that it is an inherent difference between the force-generating properties of the outer and inner arms that is responsible for the observed experimental result, and not something inherently intrinsic to either model.

The experimental evidence that provides support for the GC hypothesis was systematically collected and recently presented in an extensive review (Lindemann 2011). In the short time since that review was published, some very important studies have emerged that change the shape of the discourse on the flagellar beating mechanism. The present review attempts to assess the impact of this new information and to assimilate it into a coherent reassessment of the clutch hypothesis.

\section{Modeling progress}

The development and testing of the GC hypothesis was limited by some of the inherent features of the original computer model. The model was based on a flagellum constructed of 30 discrete segments and time marching with $0.1 \mathrm{~ms}$ iteration intervals. The physics of flagellar motion is described by a balance of the torques acting at all points along the length of the flagellum. The bending torque developed by the action of the dynein motors is always exactly balanced by the sum of the torque that results from the elastic restoring force of the structure and the torque that results from viscous drag against the external fluid. In all versions of the original GC clutch model, the balance between hydrodynamic drag torque and the total active and passive bending torque was only approximated. Newer models of flagellar motion in a well-constructed hydrodynamic environment have been developed by other investigators and are more robust in 
this regard (Smith et al. 2009, Olson et al. 2011). This has left open the possibility that the life-like simulation of ciliary and flagellar motion achieved by the various versions of the GC model might not hold up in a more rigorous model.

Two recent developments ameliorate this concern and lend additional credence to the GC mechanism. First, Brokaw (2014) implemented the GC mechanism in his modeling format and applied it to the situation of two interacting doublets. He used this model to duplicate the experimental results obtained in two doublet interactions of the Chlamydomonas axoneme that were published by Aoyama \& Kamiya (2005), discussed in more detail later in the present review. In addition, when the doublets were prevented from separating completely by the imposition of resistive elements between them, the model produced a beat cycle with propagating bends initiated at the base and moving toward the tip. This was effectively the first test of the GC mechanism in an independent modeling format and in a format that was more rigorous and mathematically sophisticated. The Brokaw model also had a representation of the dynein work cycle that was far more complete than any of the previous GC models.

The second major development was accomplished by Bayly \& Wilson (2014). They produced a continuous GC model with only the guidance of our published reports and no direct assistance. The continuous model that they developed does not have the stated limitations of the original discrete GC model. The mathematical treatment is such that it is in a form that can be readily adopted by the mathematics and physics communities. It can potentially allow for more extensive testing of the GC hypothesis and more direct comparison with alternative models. The simulations presented thus far closely resemble those of the original discrete GC model. Apparently, the liberties of approximation in the discrete model were not too great.

\section{Strange flagella, mutant flagella, and frayed flagella}

It is well documented that nature has many examples of motile flagella that are lacking one or many components of the central pair-radial spoke (cp-spoke) system. These strange flagella are often still capable of rhythmic beating and flagellar propulsion. The latest example of this is presented in a beautiful study by Mencarelli et al. (2014) for mayfly sperm. These sperm seem to retain only one type of inner-arm dynein and are missing most of the components of the cp-spoke apparatus. However, they generate functional beating and can swim. This is only the latest example of flagella that have a pared-down axoneme structure that is missing various components of the cp-radial spoke complex. The $9+0$ flagella of eel sperm are motile and have a somewhat helical beat (Woolley 1997). In the gall midge, the entire characteristic $9+2$ circular arrangement of the doublets is replaced by extravagant spirals of microtubules, and only a single row of outer-arm dyneins is present (Lupetti et al. 1998); yet the flagella beat.

Although none of this evidence alone is proof that cilia and flagella with the full complement of cp-spoke structures utilize the same mechanism to generate a beat cycle, it is certainly proof that a beat cycle can result from a mechanism that does not rely on the elements of the cp-spoke complex. It also suggests that the basic beating mechanism may depend more on conserving the spacing arrangement of the microtubules relative to the inter-doublet arrangement of dynein motors than it does on any central governing control.

In Chlamydomonas, the loss of motility in mutants that are missing elements of the radial spokes or the central pair apparatus has long been considered one of the key arguments that the cp-spoke components are essential for the basic beating mechanism. However, in Chlamydomonas that are paralyzed by such mutations, motility can often be reestablished by a suppressor mutation that alters the components of an inner-arm dynein (Porter et al. 1992, 1994). Recently, the dynein regulatory complex (drc), which has a close association with the inner-arm dyneins, has been shown to include the part of the axoneme structure that bridges the doublets and was originally called the nexin link (Heuser et al. 2009). This combined structure (drc/nexin) is believed to be important in regulating the inner-arm dyneins, but it is also important in maintaining proper inter-doublet spacing. The studies by Heuser et al. (2009) and Lin et al. (2011) found that the suppressor mutations sup-pf-3 and sup-pf-4 render the drc structurally more like that of the WT. This suggests the possibility that the suppressors may act at least partially by remediating a mechanical deficiency created by the missing components in the paralyzed mutants.

A most impressive recent study (Oda et al. 2014) demonstrated that the loss of motility in central pair projection-deficient mutants of Chlamydomonas can be restored by replacing the missing projection with an unrelated protein of suitable size. These findings on Chlamydomonas suggest that the $\mathrm{drc} /$ nexin and the radial spoke-cp interactions may both contribute mechanically to the regulation of inter-doublet spacing, and this may be their essential role in establishing a beat cycle.

There is certainly ample evidence to suggest that there are many kinases, phosphatases, and $\mathrm{Ca}^{2+}$ control elements within the intact axoneme of cilia and flagella. It has been shown that these elements are found in key locations on the spokes and in the drc/nexin and that they impact the functioning of the dynein motors (for reviews, see Porter \& Sale (2000) and Elam et al. (2009)). It is not surprising that activating and deactivating subsets of the dyneins must be necessary to turn the flagellum off at times and to alter the shape of the beat cycle for the purpose of cellular responses such as chemotaxis, phototaxis, and hyperactivation. We 
contend that it is more likely that these regulatory features act on a common underlying beat mechanism rather than constructing a novel way of generating a beat cycle from scratch.

Certain inner-arm dyneins that are subject to tight regulation may actually be important in controlling interdoublet spacing. Two of the inner-arm dyneins have been shown to be poor at translocating microtubules, but they are particularly good at hanging on to a microtubule. Specifically I1 (dynein f), the double-headed inner-arm dynein, and dynein e seem to be specialized to provide inter-doublet adhesion more so than power is. Interdoublet adhesion, or how well the dyneins hold on and resist doublet separation, is a key parameter in the GC model that regulates the 'off' switch. Dynein $\mathrm{f}$ is so crucial that it seems to be important for normal beating in Chlamydomonas and is heavily subjected to regulation (Wirschell et al. 2007). Kotani et al. (2007) found that dynein factually acts as a drag on the gliding movement of microtubules powered by other dyneins, but it is much better at holding on than other dyneins are. Studies by Toba et al. (2011) and Heuser et al. (2012) have shown that at least one of the 11 motor domains does not contribute to microtubule sliding and that the intermediate chain/light chain complex also makes contact with the neighboring doublet. Taken together, these observations suggest that the $\mathrm{I} 1$ complex may act more as a mobile spacer than as a source of torque generation.

Kubo et al. (2012) found that dynein e, which is also heavily associated with the drc/nexin, also plays a peculiar role in motility regulation. It binds more strongly to polyglutamylated tubulin, which improves the active beating of the flagellum, yet it actually slows down the rate at which the doublets disintegrate by sliding. This suggests that its role is more directed at hanging on to the adjacent microtubule than it is at generating movement. It may well be that some of the dyneins themselves play a role in maintaining interdoublet separation and resisting dissociation of the doublets. This could provide a method for regulating the t-force switch-point thresholds that govern and coordinate the power-generating dyneins.

Kamiya \& Okagaki (1986) published an excellent study on the behavior of individual pairs of doublets that interact with each other after partial disintegration of the Chlamydomonas flagellum. The doublets make contact, adhere to each other, proceed to bend, and finally release from the base to the tip. When the GC clutch was first proposed, this experiment was used as supporting evidence that dyneins could in fact provide the motive force to terminate their own action. When the doublets bend, they come under tension or compression that generates normal forces that pry the pair of doublets apart. Aoyama \& Kamiya (2005) later published a much more detailed investigation of the same phenomenon in which they cast doubt on the t-force explanation of the cycle, because many of the events did not involve obvious bending of the flagellum in the dissociation region.

The story did not end there. Brokaw (2009) conducted an in-depth physical analysis of the two-doublet experiment. He showed that with a reasonable assumption for the stiffness of an individual doublet, the buckling of the doublet that was under compression would be adequate to generate sufficient $t$-force to cause the initiation of disengagement. His computed simulations of the twodoublet experiment were an impressive match to the experimentally observed video sequences.

Finally, the two-doublet experiment with frayed Chlamydomonas axonemes was revisited by Mukundan et al. (2014) using a somewhat modified protocol for splitting the axonemes. They used low $(0.01 \mathrm{mM})$ ATP to activate the sliding of the doublets and a higher $\mathrm{Ca}^{2+}$ concentration than that used in the prior experiments. Under those conditions, they discovered that the separation of the doublets occurs after a significant pause, and during the pause, the pair of doublets is temporarily stationary and exhibits a circular curvature.

The pause and the circular curvature are surprising results. Both provide important clues about the underlying mechanism of deactivation of the dynein motors. It is the circular curvature in a static mechanical equilibrium that tells us that there must be uniform tension along most of the length of the pair of doublets. This can only be possible if all of the tension is being generated at, or near, the distal tip of the pair. The dyneins along the remainder of the pair must have switched 'off' when a threshold of curvature or t-force was reached. This is best explained by the switching mechanism proposed in the GC hypothesis. They estimated that the switching t-force was $\sim 0.2 \mathrm{nN} /$ micron. The pause that occurs before doublet separation is even more interesting, because it can only occur if the dyneins are still hanging on to their binding sites on the adjacent tubule (Lindemann 2014). In the frayed axoneme, there are no permanent inter-doublet connections; consequently, the dyneins, or at least a subset of them, must still be resisting the separation of the doublets even after they have turned off.

\section{Seeing is believing}

The results from the study by Mukundan et al. (2014) tell us that the dyneins (or some of them) can remain physically attached to the adjacent doublet and yet be turned off as far as the generation of bending torque is concerned. This is not consistent with the original GC mechanism. In the original conception of the GC mechanism, it was assumed that the dyneins are turned off when they are physically released from the adjacent doublet. Nevertheless, the Mukundan et al. (2014) results are consistent with the images of dyneins in intact axonemes.

It has been consistently observed that the dynein stalks appear to stay connected to the B-subtubule of the 
adjacent doublet in the rigor, relaxation, and active states. This pattern was first evident in elegant freeze fracture replicas on both rigor and relaxed flagella (Goodenough \& Heuser 1982, 1985) and was later reinforced by images on cryofixed active axonemes (Burgess 1995). The latest images from cryoelectron tomography reconstructions are also arriving at the same ultrastructural picture (Oda et al. 2007, Kikkawa 2013, Oda \& Kikkawa 2013, Lin et al. 2014).

Can this accumulating evidence be reconciled with the GC hypothesis? The ultrastructural evidence from electron micrographic studies suggests that dyneins may actually be attached to the adjacent B-subtubule almost all of the time. The dynein stalk is only in the detached state briefly as the site of attachment 'jumps' after the power stroke. The cryo-reconstructions suggest that the attachments of the dynein stalk to the B-subtubule persist in the low-affinity, relaxed condition. In the original conception of the GC hypothesis, the gap between the doublets in the relaxed condition was thought to be too great for dynein attachment. Mechanical studies of flagellar stiffness seemed to support this view. When flagella are in the relaxed condition, which is induced by either vanadate inhibition of the dynein (Lindemann et al. 2005, Pelle et al. 2009) or inhibition of dynein by $10 \mathrm{mM}$ ATP (Lindemann et al. 1973), the flagella are in their most flexible state and their stiffness is minimized.

If the dyneins are still bridging the doublets in the relaxed condition, as the electron micrographic evidence suggests, then it must be that dyneins in the lowaffinity state are free to glide from binding site to binding site along the B-subtubule with little resistance to shear displacement. The study by Mukundan et al. (2014) not only supports this interpretation, but it also suggests that even when this is the case, the power cycle of the dynein is terminated when curvature or $t$-force reaches a certain threshold.

How might this be accomplished mechanistically? Goodenough \& Heuser (1982) showed that in the rigorlike state, the globular heads of the dynein arms were nested in a complex of densities on the A-subtubule. They also showed that in the relaxed condition, this nesting pattern was absent and the globular heads were not as tightly associated with the dense structures on the A-subtubule. Figure 4 illustrates the differences between the rigor and relaxed configurations based on the Goodenough \& Heuser images. The figure also illustrates that if the length of the stalk of the dynein is conserved, then the inter-doublet spacing must, of necessity, be less in the rigor state. Burgess (1995) later showed that the same rigor and relaxed configurations that were seen by Goodenough \& Heuser were intermixed in images from cryofixed actively motile flagella. The latest work by Lin et al. (2014) may also be viewed as being generally consistent with Burgess's earlier results.

Based on these images, it is reasonable to propose the following explanation: it may be that the nested, or
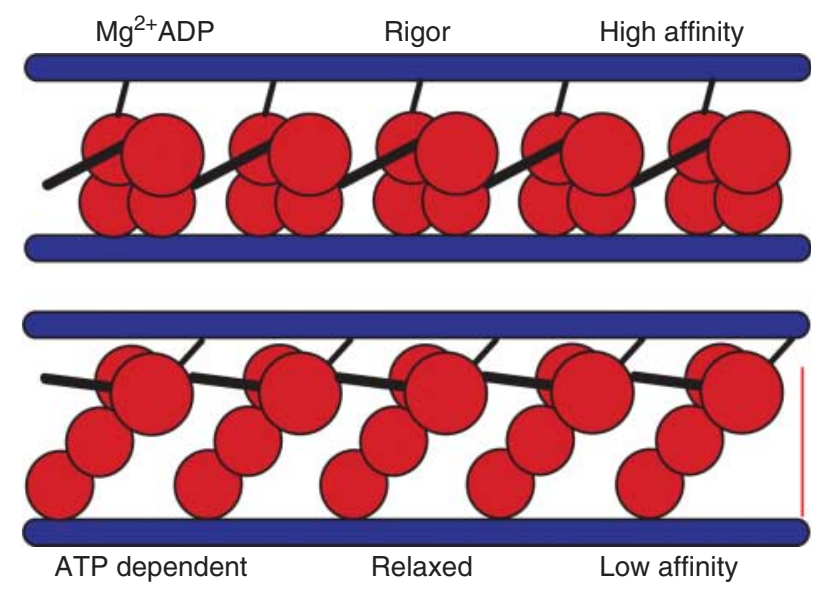

Figure 4 Rigor and relaxed configurations of dynein. This is a representation of the differences in configuration of the outer-arm dyneins that are consistent with the freeze-fracture gold replicas of axonemes in the rigor and relaxed states published by Goodenough \& Heuser (1982). It also shows that the nesting of the dynein head on the A-subtubule requires that the inter-doublet spacing is reduced in the rigor state if the length of the dynein stalk is conserved. A reduction in the inter-doublet spacing that accompanies the formation of rigor bridges has been documented by Zanetti et al. (1979).

rigor-like, configuration is a required step in the complete cross-bridge cycle of power generation. This is directly consistent with the proposal made by Burgess (1995) from his micrographs of active flagella. It logically follows that if the nested, rigor-like step is prohibited by the separation of the doublets, then it may also terminate the ability of the dynein to deliver force between the outer doublets. Stated another way, if the power stroke of the cross-bridge cycle is interrupted by spatial constraints, motive force can no longer be generated. When the technique of cryoelectron tomography is eventually employed to look at whole actively beating axonemes, it may be possible to determine if inter-doublet spacing varies with the location and phase of the beat cycle.

An additional factor to consider is that whenever flagella are actively beating, ATP is always present to bind to the dynein head and displace ADP. ATP binding to the dynein regulatory sites is likely the trigger that puts the dynein stalk into the low-affinity state, as has been postulated previously (Porter \& Johnson 1983). Blockade of the rigor-like step in the power stroke and conversion of the stalk connection to the low-affinity state could effectively terminate shear force transmission between the doublets. This potential mechanism is illustrated in Fig. 5.

Following an analysis of the flexibility of the dynein stem, we proposed that force transmission between the doublets would be severely limited if there were not some mechanism to stabilize the dynein head during the power stroke (Lindemann \& Hunt 2003). In addition, work from Warner's laboratory (Warner \& Mitchell 1978, Zanetti et al. 1979) showed beyond any doubt that the formation of rigor bridges physically reduces the size of 

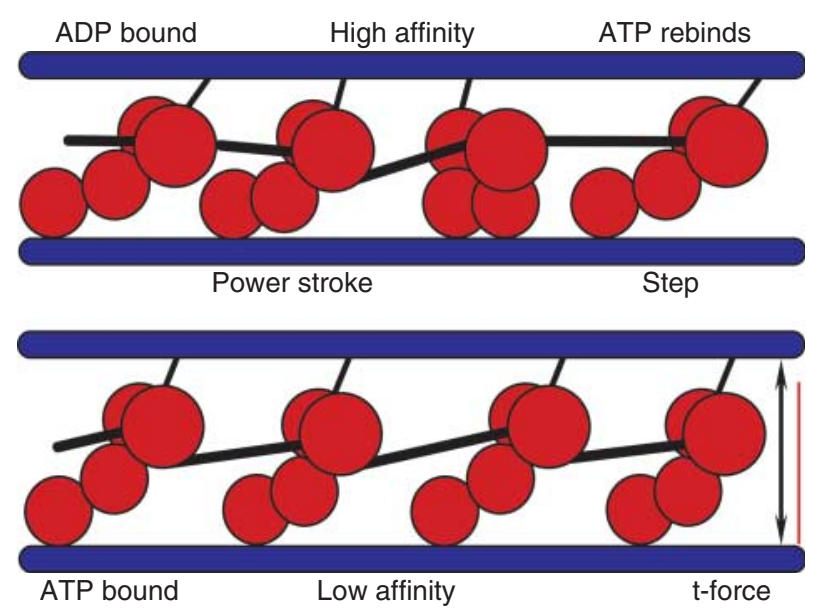

Figure 5 The proposed effect of doublet spacing on the dynein power cycle. It has been suggested that the rigor configuration of the dynein bridge alternates with the relaxed configuration when dynein is in the active state that generates flagellar motility (Burgess 1995). This is illustrated in the upper panel using representations of the dynein crossbridge cycle that include both the relaxed, low-affinity dynein state and the rigor, high-affinity state. Force transmission between the doublets occurs when the dynein is in the high-affinity, rigor-like configuration. This configuration corresponds to the power stroke of the motor. The lower panel illustrates how increased doublet separation interferes with the formation of the high-affinity, rigor-like state and therefore interferes with the completion of the power cycle. The presence of ATP during the motile condition ensures that the dynein active sites have bound ATP and revert to the relaxed, low-affinity state. Therefore, increased interdoublet spacing may render dyneins incapable of force generation.

the axoneme by drawing the doublets together. They also showed that the rigor bridged state is facilitated by the divalent ions $\mathrm{Mg}^{2+}$ and $\mathrm{Ca}^{2+}$. We now know that the reduction in inter-doublet spacing in rigor cannot be dependent on the attachment of the dynein stalk to the B-subtubule, because that attachment is visualized in both the rigor and the relaxation states. So what pulls the doublets together and reduces the axoneme diameter in the rigor state? It must be an interaction facilitated at another location in the dynein bridge!

The length of the coiled coil stalk of the dynein heavy chain is $\sim 15 \mathrm{~nm}$ (Burgess et al. 2003). Changes in doublet spacing would have to be accommodated by other portions of the heavy chain, seeing as protein coiled coils are relatively linear and inextensible. In the rigor state, this could be accomplished by altering the nesting of the globular head in the protein densities on the A-subtubule of the outer doublet (as was suggested by the Goodenough \& Heuser (1982) images). This possibility is illustrated in Fig. 5.

Alternatively, t-force could cause a change in the folding structure of the globular head. In support of this, we can consider Fig. 28 in the Goodenough \& Heuser (1982) study, wherein they show a 'stressed' relaxed axoneme. The stalk attachments to the B-subtubule of many of the dyneins had released, whereas other dyneins were greatly stretched and still others showed the globular portion of the dynein suspended between the doublets at a greater distance from the A-tubule than normal. If the doublet spacing were to be increased by $\mathrm{t}$-force and the stalk remained attached, it would most likely be accommodated by an uncoiling of the folded AAA portion of the heavy chain and linker. In fact, Kikkawa (2013) elaborated on this possibility in a recent review on the regulation of dynein and suggested that dynein may be designed to act as a tension sensor. He proposed that the gap between AAA1 and AAA2 is strain-dependent, and the positioning of the two domains may be crucial for ATP hydrolysis in the power cycle. Either one of these putative mechanisms could potentially disrupt the power stroke by effectively preventing the formation of the dynein configuration that is required to carry out the power stroke of the dynein cross-bridge cycle.

Given the accumulated evidence, we now have reason to believe that a required configuration of the dynein heavy chain is needed for the force generation step in the cross-bridge cycle. If that configuration is prohibited by inter-doublet spacing, then the crossbridge cycle cannot generate the necessary shearing force to make the doublets slide. Although this is clearly an unexpected modification of the original conception of the GC mechanism, it is still consistent with a beat cycle controlled by $\mathrm{t}$-force and inter-doublet spacing. If this revised view of the mechanics of the GC mechanism is correct, then factors that have been shown to stabilize the rigor configuration of the dynein bridge, such as $\mathrm{Mg}^{2+}$ and $\mathrm{Ca}^{2+}$ concentration, should directly impact the parameters of the beat cycle by regulating the stability of the required power stroke step of the dynein force-producing cycle.

The most recent experiments from our laboratory seem to support this interpretation. We discovered that a large excess of ATP un-complexed with $\mathrm{Mg}^{2+}$ and a large excess of $\mathrm{Mg}^{2+}$ un-complexed with ATP are both incompatible with stable beating when we used reactivated bull sperm to map the ranges of $\mathrm{Mg}^{2+}$ and ATP that are compatible with a stable beat cycle (Lindemann et al. 2014). In addition, we were able to show that a high $\mathrm{Mg}^{2+}$ condition reduces the axoneme diameter and inter-doublet spacing (Lesich et al. 2014). This was predicted by Warner's (1978) earlier deduction that $\mathrm{Mg}^{2+}$ stabilizes rigor bridges, and this is what draws the doublets closer together. In support of this explanation, we also observed that a high $\mathrm{Mg}^{2+}$ condition renders the flagella stiffer, and a high ATP condition renders the flagellum flaccid.

These results are all compatible with the hypothesis that excess $\mathrm{Mg}^{2+}$ favors the formation of rigor bridges even in the presence of some ATP and that excess ATP prevents effective power generation by preventing the high-affinity state that is needed for the force transmission between the doublets. If indeed the dynein stalk remains attached to the B-subtubule under both 
conditions, the only explanation for a reduction in doublet spacing in a high $\mathrm{Mg}^{2+}$ state is that $\mathrm{Mg}^{2+}$ induces a change in another part of the dynein arm assemblage. It follows that this configuration change must be what pulls the doublets closer in rigor. Further experiments to test this prediction of the modified GC hypothesis should be informative.

\section{Declaration of interest}

The authors declare that there is no conflict of interest that could be perceived as prejudicing the impartiality of the review.

\section{Funding}

This research did not receive any specific grant from any funding agency in the public, commercial or not-for-profit sector.

\section{References}

Aoyama S \& Kamiya R 2005 Cyclical interactions between two outer doublet microtubules in split flagellar axonemes. Biophysical Journal 89 3261-3268. (doi:10.1529/biophysj.105.067876)

Bayly PV \& Wilson KS 2014 Equations of interdoublet separation during flagella motion reveal mechanisms of wave propagation and instability. Biophysical Journal 107 1756-1772. (doi:10.1016/j.bpj.2014.07.064)

Brokaw CJ 1999 Computer simulation of flagellar movement: VII. Conventional but functionally different cross-bridge models for inner and outer arm dyneins can explain the effects of outer arm dynein removal. Cell Motility and the Cytoskeleton 42 134-148. (doi:10.1002/ (SICI)1097-0169(1999)42:2 <134::AID-CM5 > 3.0.CO;2-B)

Brokaw CJ 2009 Simulation of cyclic dynein-driven sliding, splitting, and reassociation in an outer doublet pair. Biophysical Journal 97 2939-2947. (doi:10.1016/j.bpj.2009.09.022)

Brokaw CJ 2014 Computer simulation of flagellar movement X: doublet pair splitting and bend propagation modeled using stochastic dynein kinetics. Cytoskeleton 71 273-284. (doi:10.1002/cm.21168)

Brokaw CJ \& Kamiya R 1987 Bending patterns of Chlamydomonas flagella: IV. Mutants with defects in inner and outer dynein arms indicate differences in dynein arm function. Cell Motility and the Cytoskeleton 8 68-75. (doi:10.1002/cm.970080110)

Burgess SA 1995 Rigor and relaxed outer dynein arms in replicas of cryofixed motile flagella. Journal of Molecular Biology 250 52-63. (doi:10.1006/jmbi.1995.0357)

Burgess SA, Walker ML, Sakakibara H, Knight PJ \& Oiwa K 2003 Dynein structure and power stroke. Nature 421 715-718. (doi:10.1038/ nature01377)

Davis EE, Brueckner M \& Katsanis N 2006 The emerging complexity of the vertebrate cilium: new functional roles for an ancient organelle. Developmental Cell 11 9-19. (doi:10.1016/j.devcel.2006.06.009)

Dutcher SK 1995 Flagellar assembly in two hundred and fifty easy-to-follow steps. Trends in Genetics 11 398-404. (doi:10.1016/S0168-9525(00)89123-4)

Elam CA, Sale WS \& Wirschell M 2009 The regulation of dynein-driven microtubule sliding in Chlamydomonas flagella by axonemal kinases and phosphatases. Methods in Cell Biology 92 133-151. (doi:10.1016/ 50091-679X(08)92009-4)

Gatti JL, King SM, Moss AG \& Witman GB 1989 Outer arm dynein from trout spermatozoa. Purification, polypeptide composition, and enzymatic properties. Journal of Biolgical Chemistry 264 11450-11457.

Goodenough UW \& Heuser JE 1982 Substructure of the outer dynein arm. Journal of Cell Biology 95 798-815. (doi:10.1083/jcb.95.3.798)

Goodenough UW \& Heuser JE 1985 Substructure of inner dynein arms, radial spokes, and the central pair/projection complex of cilia and flagella. Journal of Cell Biology 100 2008-2018. (doi:10.1083/jcb.100.6. 2008)
Heuser T, Raytchev M, Krell J, Porter ME \& Nicastro D 2009 The dynein regulatory complex is the nexin link and a major regulatory node in cilia and flagella. Journal of Cell Biology 187 921-933. (doi:10.1083/jcb. 200908067)

Heuser T, Barber CF, Lin J, Krell J, Rebesco M, Porter ME \& Nicastro D 2012 Cryoelectron tomography reveals doublet-specific structures and unique interactions in the 11 dynein. PNAS 109 E2067-E2076. (doi:10.1073/pnas.1120690109)

Holcomb-Wygle DL, Schmitz KA \& Lindemann CB 1999 Flagellar arrest behavior predicted by the geometric clutch model is confirmed experimentally by micromanipulation experiments on reactivated bull sperm. Cell Motility and the Cytoskeleton 44 177-189. (doi:10.1002/ (SICI)1097-0169(199911)44:3<177::AID-CM3 > 3.0.CO;2-W)

Hom EF, Witman GB, Harris EH, Dutcher SK, Kamiya R, Mitchell DR, Pazour GJ, Porter ME, Sale WS, Wirschell M etal. 2011 A unified taxonomy for ciliary dyneins. Cytoskeleton 68 555-565. (doi:10.1002/cm.20533)

Kamiya R \& Okagaki T 1986 Cyclical bending of two outer-doublet microtubules in frayed axonemes of Chlamydomonas. Cell Motility and the Cytoskeleton 6 580-585. (doi:10.1002/cm.970060606)

Kamiya R \& Yagi T 2014 Functional diversity of axonemal dyneins as assessed by in vitro and in vivo motility assays of chlamydomonas mutants. Zoological Science 31 633-644. (doi:10.2108/zs140066)

Kikkawa M 2013 Big steps toward understanding dynein. Journal of Cell Biology 202 15-23. (doi:10.1083/jcb.201304099)

Kotani N, Sakakibara H, Burgess SA, Kojima H \& Oiwa K 2007 Mechanical properties of inner-arm dynein- $f$ (dynein I1) studied with in vitro motility assays. Biophysical Journal 93 886-894. (doi:10.1529/biophysj.106. 101964)

Kubo T, Yagi T \& Kamiya R 2012 Tubulin polyglutamylation regulates flagellar motility by controlling a specific inner-arm dynein that interacts with the dynein regulatory complex. Cytoskeleton 69 1059-1068. (doi:10.1002/cm.21075)

Lesich KA, DePinho TG, Dang L \& Lindemann CB 2014 Ultrastructural evidence that motility changes caused by variations in ATP, $\mathrm{Mg}$, and ADP correlate to conformational changes in reactivated bull sperm axonemes. Cytoskeleton 71 649-661. (doi:10.1002/cm.21199)

Lin J, Tritschler D, Song K, Barber CF, Cobb JS, Porter ME \& Nicastro D 2011 Building blocks of the nexin-dynein regulatory complex in Chlamydomonas flagella. Journal of Biological Chemistry 286 29175-29191. (doi:10.1074/jbc.M111.241760)

Lin J, Okada K, Raytchev M, Smith MC \& Nicastro D 2014 Structural mechanism of the dynein power stroke. Nature Cell Biology 16 479-485. (doi:10.1038/ncb2939)

Lindemann CB 1994a A "Geometric Clutch" hypothesis to explain oscillations of the axoneme of cilia and flagella. Journal of Theoretical Biology 168 175-189. (doi:10.1006/jtbi.1994.1097)

Lindemann CB 1994b A model of flagellar and ciliary functioning which uses the forces transverse to the axoneme as the regulator of dynein activation. Cell Motility and the Cytoskeleton 29 141-154. (doi:10.1002/ cm.970290206)

Lindemann CB 2002 Geometric clutch model version 3: the role of the inner and outer arm dyneins in the ciliary beat. Cell Motility and the Cytoskeleton 52 242-254. (doi:10.1002/cm.10049)

Lindemann CB 2011 Experimental evidence for the geometric clutch hypothesis. Current Topics in Developmental Biology 95 1-31. (doi:10. 1016/B978-0-12-385065-2.00001-3)

Lindemann CB 2014 Dynein regulation: going into circles can set things straight. Biophysical Journal 106 2285-2287. (doi:10.1016/j.bpj.2014. 04.003)

Lindemann CB \& Hunt AJ 2003 Does axonemal dynein push, pull or oscillate? Cell Motility and the Cytoskeleton 56 237-244. (doi:10.1002/ cm.10148)

Lindemann CB \& Kanous KS 1995 "Geometric clutch" hypothesis of axonemal function: key issues and testable predictions. Cell Motility and the Cytoskeleton 31 1-8. (doi:10.1002/cm.970310102)

Lindemann CB \& Lesich KA 2010 Flagellar and ciliary beating: the proven and the possible. Journal of Cell Science 123 519-528. (doi:10.1242/jcs. 051326)

Lindemann CB, Rudd WG \& Rikmenspoel R 1973 The stiffness of the flagella of impaled bull sperm. Biophysical Journal 13 437-448. (doi:10.1016/S0006-3495(73)85997-1) 
Lindemann CB, Macauley LJ \& Lesich KA 2005 The counterbend phenomenon in dynein-disabled rat sperm flagella and what it reveals about the interdoublet elasticity. Biophysical Journal 89 1165-1174. (doi:10.1529/biophysj.105.060681)

Lindemann CB, DePinho TG \& Lesich KA 2014 The physiological role of $\mathrm{ADP}$ and $\mathrm{Mg}$ in maintaining a stable beat cycle in bull sperm. Cytoskeleton 71 638-648. (doi:10.1002/cm.21200)

Lupetti P, Mencarelli C, Rosetto M, Heuser JE \& Dallai R 1998 Structural and molecular characterization of dynein in a gall-midge insect having motile sperm with only the outer arm. Cell Motility and the Cytoskeleton 39 303-317. (doi:10.1002/(SICI)1097-0169(1998)39:4<303::AID$\mathrm{CM}$ 5 $>3.0 . \mathrm{CO} ; 2-3)$

Mencarelli C, Mercati D, Dallai R \& Lupetti P 2014 Ultrastructure of the sperm axoneme and molecular analysis of axonemal dynein in Ephemeroptera (Insecta). Cytoskeleton 71 328-339. (doi:10.1002/cm.21175)

Mukundan V, Sartori P, Geyer VF, Julicher F \& Howard J 2014 Motor regulation results in distal forces that bend partially disintegrated Chlamydomonas axonemes into circular arcs. Biophysical Journal 106 2434-2442. (doi:10.1016/j.bpj.2014.03.046)

Oda T \& Kikkawa M 2013 Novel structural labeling method using cryoelectron tomography and biotin-streptavidin system. Journal of Structural Biology 183 305-311. (doi:10.1016/j.jsb.2013.07.003)

Oda T, Hirokawa N \& Kikkawa M 2007 Three-dimensional structures of the flagellar dynein-microtubule complex by cryoelectron microscopy. Journal of Cell Biology 177 243-252. (doi:10.1083/jcb.200609038)

Oda T, Yagi T, Yanagisawa H \& Kikkawa M 2013 Identification of the outerinner dynein linker as a hub controller for axonemal dynein activities. Current Biology 23 656-664. (doi:10.1016/j.cub.2013.03.028)

Oda T, Yanagisawa H, Yagi T \& Kikkawa M 2014 Mechanosignaling between central apparatus and radial spokes controls axonemal dynein activity. Journal of Cell Biology 204 807-819. (doi:10.1083/jcb. 201312014)

Olson SD, Suarez SS \& Fauci LJ 2011 Coupling biochemistry and hydrodynamics captures hyperactivated sperm motility in a simple flagellar model. Journal of Theoretical Biology 283 203-216. (doi:10.1016/j.jtbi.2011.05.036)

Paschal BM, King SM, Moss AG, Collins CA, Vallee RB \& Witman GB 1987 Isolated flagellar outer arm dynein translocates brain microtubules in vitro. Nature 330 672-674. (doi:10.1038/330672a0)

Pazour GJ, Agrin N, Leszyk J \& Witman GB 2005 Proteomic analysis of a eukaryotic cilium. Journal of Cell Biology 170 103-113. (doi:10.1083/ jcb.200504008)

Pelle DW, Brokaw CJ, Lesich KA \& Lindemann CB 2009 Mechanical properties of the passive sea urchin sperm flagellum. Cell Motility and the Cytoskeleton 66 721-735. (doi:10.1002/cm.20401)
Porter ME \& Johnson KA 1983 Characterization of the ATP-sensitive binding of Tetrahymena $30 \mathrm{~S}$ dynein to bovine brain microtubules. Journal of Biological Chemistry 258 6575-6581.

Porter ME \& Sale WS 2000 The $9+2$ axoneme anchors multiple inner arm dyneins and a network of kinases and phosphatases that control motility. Journal of Cell Biology 151 F37-F42. (doi:10.1083/jcb.151.5.F37)

Porter ME, Power J \& Dutcher SK 1992 Extragenic suppressors of paralyzed flagellar mutations in Chlamydomonas reinhardtii identify loci that alter the inner dynein arms. Journal of Cell Biology 118 1163-1176. (doi:10.1083/jcb.118.5.1163)

Porter ME, Knott JA, Gardner LC, Mitchell DR \& Dutcher SK 1994 Mutations in the SUP-PF-1 locus of Chlamydomonas reinhardtii identify a regulatory domain in the $\beta$-dynein heavy chain. Journal of Cell Biology 126 1495-1507. (doi:10.1083/jcb.126.6.1495)

Schmitz KA, Holcomb-Wygle DL, Oberski DJ \& Lindemann CB 2000 Measurement of the force produced by an intact bull sperm flagellum in isometric arrest and estimation of the dynein stall force. Biophysical Journal 79 468-478. (doi:10.1016/S0006-3495(00)76308-9)

Smith DJ, Gaffney EA, Gadelha H, Kapur N \& Kirkman-Brown JC 2009 Bend propagation in the flagella of migrating human sperm, and its modulation by viscosity. Cell Motility and the Cytoskeleton 66 220-236. (doi:10.1002/cm.20345)

Toba S, Fox LA, Sakakibara H, Porter ME, Oiwa K \& Sale WS 2011 Distinct roles of $1 \alpha$ and $1 \beta$ heavy chains of the inner arm dynein 11 of Chlamydomonas flagella. Molecular Biology of the Cell 22 342-353. (doi:10.1091/mbc.E10-10-0806)

Warner FD 1978 Cation-induced attachment of ciliary dynein crossbridges. Journal of Cell Biology 77 R19-R26. (doi:10.1083/jcb.77.3.R19)

Warner FD \& Mitchell DR 1978 Structural conformation of ciliary dynein arms and the generation of sliding forces in Tetrahymena cilia. Journal of Cell Biology 76 261-277. (doi:10.1083/jcb.76.2.261)

Wirschell M, Hendrickson T \& Sale WS 2007 Keeping an eye on 11: I1 dynein as a model for flagellar dynein assembly and regulation. Cell Motility and the Cytoskeleton 64 569-579. (doi:10.1002/cm.20211)

Woolley DM 1997 Studies on the eel sperm flagellum. The structure of the inner dynein arm complex. Journal of Cell Science 110 85-94.

Zanetti NC, Mitchell DR \& Warner FD 1979 Effects of divalent cations on dynein cross bridging and ciliary microtubule sliding. Journal of Cell Biology 80 573-588. (doi:10.1083/jcb.80.3.573)

Received 24 September 2014

First decision 12 November 2014

Revised manuscript received 23 April 2015

Accepted 24 April 2015 